\title{
Upon Awakening
}

National Cancer Institute

\section{Source}

National Cancer Institute. Upon Awakening. NCI Thesaurus. Code C64921.

Used to indicate that an event is to commence following the end of sleeping. 